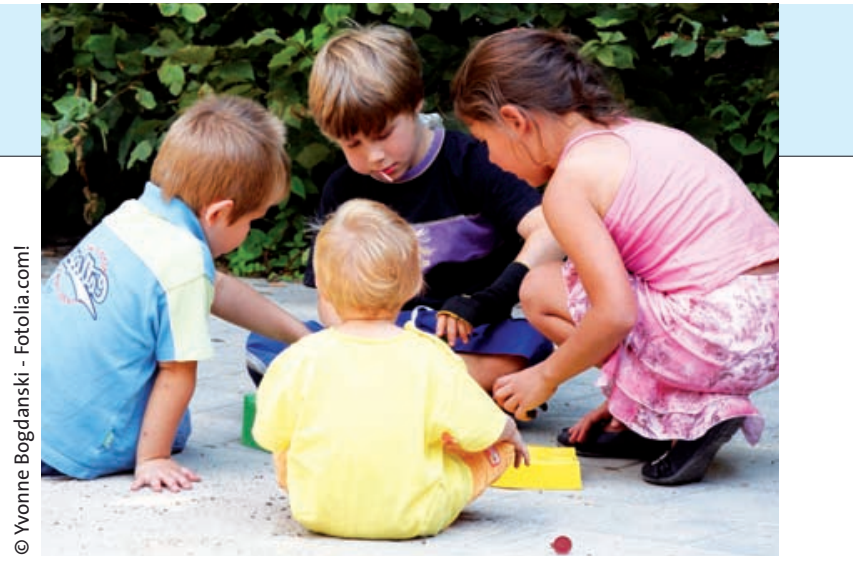

\title{
Hygiene-Hypothese mit umgekehrtem Vorzeichen
}

Der frühe Kontakt mit Mikroorganismen soll eine protektive Wirkung gegen allergische Erkrankungen haben. Eine deutsche Analyse weist jedoch darauf hin, dass bei Vorliegen einer Filaggrin-Mutation genau das Gegenteil der Fall sein kann.

C in deutsches Autorenteam wertete Daten der beiden Kohortenstudien LISAplus und GINIplus aus. Analysiert wurden die Kinder, bei denen eine Testung auf Filaggrin-Mutationen durchgeführt worden war $(\mathrm{n}=$ 2.867). Bei $6,7 \%$ (LISAplus) und 6,0\% (GINIplus) waren diese Mutationen nachweisbar. Mit der Auswertung sollte geklärt werden, ob die Anwesenheit älterer Geschwister (und der damit postulierte vermehrte Kontakt mit Keimen) einen protektiven Effekt auf die Entwicklung von Ekzemen zeigt.

Ältere Geschwister hatten nach diesen Daten keinen signifikanten Einfluss auf die Entwicklung von Ekzemen: Die entsprechende Odds Ratio (OR) betrug bei den Teilnehmern der LISAplus-Studie 1,21 (95\%-Konfidenzintervall, CI: 0,93-1,58). Für Studienteilnehmer, die keine Kindertagesstätten besuchten, lag das Risiko bei 1,01 (CI: 0,74-1,37). In der GINIplus-Studie ergaben sich Werte von 1,00 (CI: 0,81-1,24) bzw. 1,00 (CI: 0,80-1,24).

Bei gleichzeitigem Vorliegen einer Filaggrin-Mutation und der Anwesenheit älterer Geschwister lag das Ekzemrisiko in der LISAplus-Studie bei 1,51 (CI: 0,62-3,68, keine Signifikanz). In der GINIplus-Studie zeigte sich hier ein signifikant erhöhtes Risiko von 2,38 (CI: 1,09-5,17). Bei den Kindern, die keine Kindertagesstätten besuchten, war in beiden Studien eine signifikante Zunahme des Ekzemrisikos nachweisbar (OR: 3,27, CI: 1,14-9,36 bzw. OR: 2,41, CI: 1,06-5,48).

Fazit: In dieser Analyse konnte der in früheren Studien gezeigte protektive Effekt von älteren Geschwistern auf das Ekzemrisiko nicht bestätigt werden. Bei einer nachgewiesenen Filaggrin-Mutation erhöhte die Anwesenheit von Geschwistern sogar das Risiko. 